\title{
Overweight and obesity are associated with decreased magnesium intake in people with asthma
}

by Alexandra G. Kazaks and Judith S. Stern

Asthma is a serious health problem that is more prevalent among lowincome persons. The risk of asthma and the severity of its symptoms may be increased by the low dietary intake of magnesium and other nutrients that protect against asthma, coupled with the high energy intakes that result in overweight and obesity. This study compared people with asthma to healthy controls, and showed that total body magnesium stores decreased with increasing weight, as measured by body mass index (BMI). Replacing low-magnesium foods with high-magnesium foods may be a practical, low-cost way to help reduce the risk of obesity and low magnesium status in people with asthma, especially in at-risk, low-income groups.

\section{$A^{s}$} sthma is a major cause of illness and disability in the United States, and is a significant public health burden. In 2003, asthma affected an estimated 20 million individuals in the United States. In 2004, the estimated annual costs due to asthma were more than $\$ 16$ billion, including the direct costs of physician and hospital visits, and medications, as well as the indirect costs of lost work days and earnings (ALA 2005).

Although asthma affects Americans of all ages, races and ethnic groups, the Centers for Disease Control and Prevention reports that low-income and minority populations experience significantly higher rates of fatalities, hospital admissions and emergency room visits (CDC 2000). While the reasons for these disparities are not clearly understood, factors such as limited access to medical care and lack of self-management skills all contribute to increased asthma symptoms.

The current concept of asthma has shifted from a disease of smooth muscle

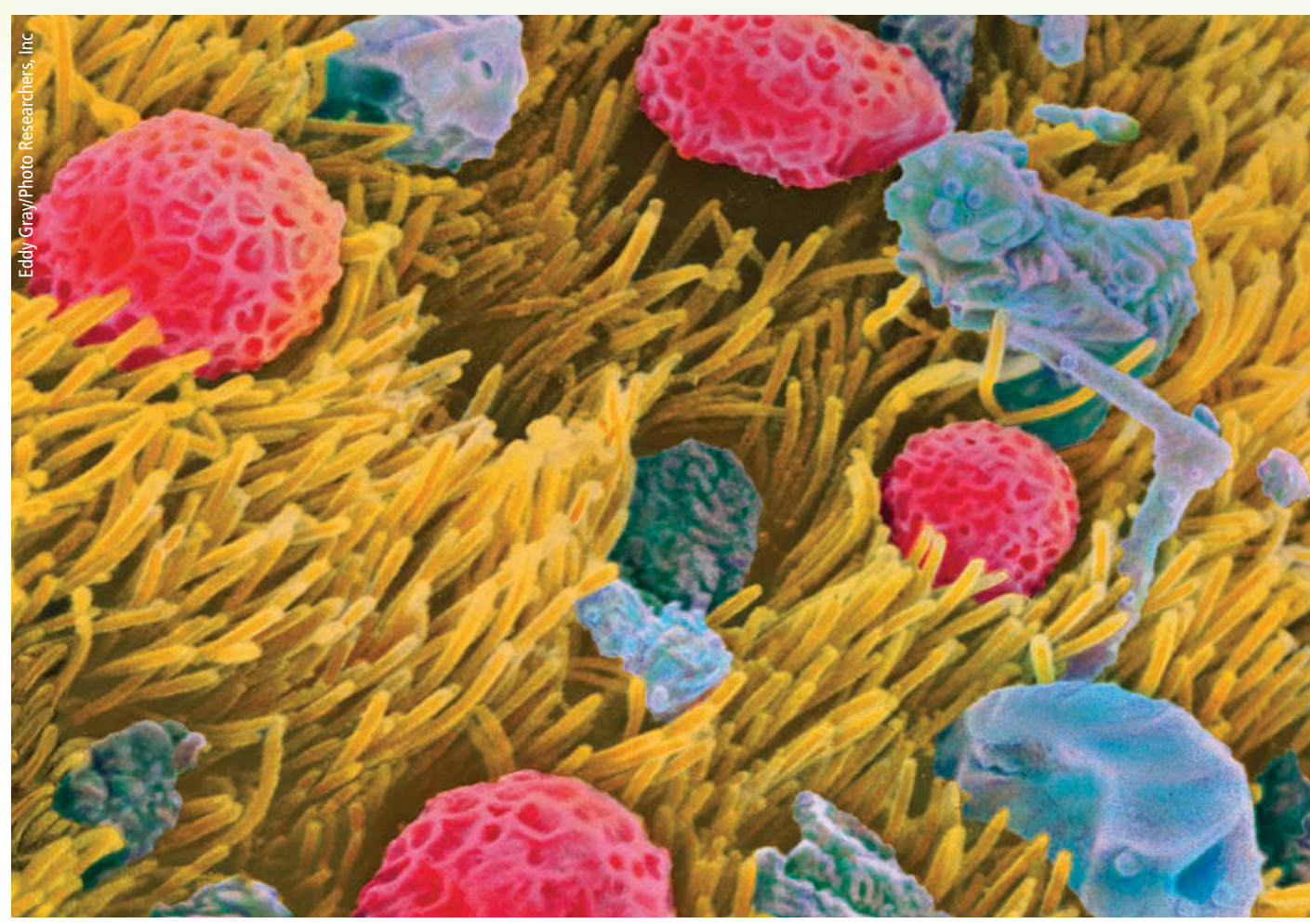

The surface of a person's windpipe (photographed with a colored electron-scanning microscope) can trap pollen grains (pink) on hairlike cilia (yellow). In asthmatic or allergic persons, the particles can cause a hypersensitive reaction that leads to breathing difficulties.

dysfunction to one characterized by the persistent inflammation of lung tissues (fig. 1). Asthma is characterized by episodes of reversible narrowing of the airways. The episodes can vary from mild chest tightness, shortness of breath, and coughing or wheezing, to a lifethreatening inability to breathe (NHLBI 2002). Asthma occurs in people who are predisposed to develop the disease due to genetic and environmental factors. A wide variety of triggers may start or worsen an asthma attack, including exposure to allergens, viral respiratory infections, airway irritants such as tobacco smoke and environmental pollutants, and exercise.

In addition, diet can have a significant effect on asthma. Asthma is characterized by an oxidant-antioxidant imbalance, and dietary intervention may shift this imbalance by preventing the formation of free radicals, making oxidants less toxic and modulating the immune response in the lung (Hartert and Peebles 2001). Any molecule can become

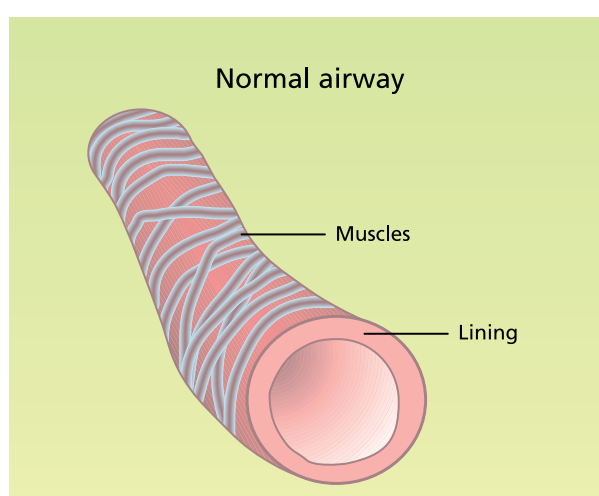

Airway in person with asthma

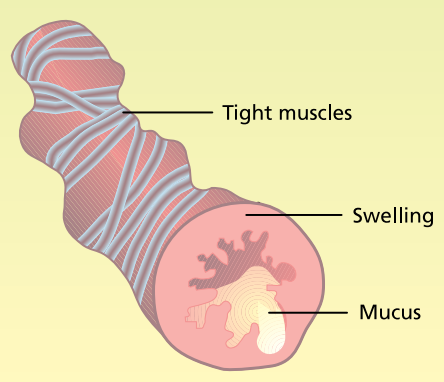

Fig. 1. Asthma pathology. Source: NHLBI 2006. 
a free radical by either losing or gaining an electron in normal reactions that occur in the body. Molecules containing these uncoupled electrons are very reactive as they try to capture or donate electrons. The body's defense systems produce antioxidants that neutralize free radicals and protect cells from damage. Antioxidants are also contributed by the diet; there are more than 4,000 compounds that act as antioxidants in foods, including vitamins C, E and magnesium. The relative importance of individual nutrients in asthma control is unclear, but magnesium may be significant (Mathew and Altura 1991).

\section{Treating asthma with magnesium}

Current therapies for asthma have limitations. First, there is no known cure; even with treatment, chronic symptoms remain a problem. Common medications for asthma include inhaled corticosteroids, and inhaled or oral beta 2-agonists. Beta-agonists are medications that relax the muscles around airways that tighten during an asthma attack, and corticosteroids are drugs that decrease inflammation within the breathing passages. These are used on a regular basis to control chronic symptoms and prevent attacks. However, these medications can be associated with adverse side effects such as cardiac arrhythmia and other cardiovascular disturbances, causing people with asthma to limit the dosage and frequency of medication use (NHLBI 2002).

Magnesium is an essential mineral with several dietary sources that is found in a variety of foods, including whole grains, green leafy vegetables, legumes and nuts. (Water, if it is high in dissolved minerals, can also be a source of magnesium.) The idea that magnesium could be useful for asthma treatment was first published in 1940, as anecdotal reports describing the beneficial effects of intravenous (IV) magnesium sulphate given during acute asthma attacks (Haury 1940). However, more recent studies assessing magnesium therapy in asthma have given conflicting results. Some studies with IV magnesium suggest that it could be helpful in acute asthma treatment (Cheuk et al. 2005), although other studies show no effect (Bernstein et al. 1995). In addition, while inhaled aerosol magnesium-sulphate improved asthma symptoms in a double-randomized, controlled trial (Rolla et al. 1987), magnesium administered in aerosol form had no effect in another similar study (Chande and Skoner 1992).

These studies suggest that IV and inhaled magnesium may improve pulmonary function in acute asthma, but are not useful in patients with more moderate episodes. It is unclear whether magnesium administration has a specific pharmacologic effect or whether it corrects an underlying deficiency. Certain groups of people, including those with asthma, may not be obtaining adequate dietary magnesium and therefore have low magnesium stores (Britton et al. 1994).

\section{Dietary magnesium and asthma risk}

Among the dietary variables associated with an increased risk of asthma is the low consumption of vegetables, milk and minerals, including magnesium. Large, population-based epidemiologic studies have found that lower dietary magnesium intakes were associated with impaired lung function, bronchial hyperreactivity and the increased risk of wheezing (Britton et al. 1994).

Numerous studies show a relationship between low magnesium intake and asthma symptoms, and indicate that intakes of magnesium in the general population do not reach recommended levels. Less than half of American adults consumed the Recommended Daily Allowance (RDA) of magnesium, according to the National Health and Nutrition Examination Survey (NHANES) 19992000. The average intake of magnesium by women 19 to 50 years of age was only $74 \%$ of the RDA. Men of the same age consumed about $94 \%$ of the RDA; in general men consume more food, so they get more magnesium overall.

Due to the inflammation associated with asthma, immune cells in lung tis- sues generate inflammatory mediators and cytokines (Barnes 2004) that then circulate throughout the body and may be a source of low-grade systemic inflammation (which can be identified with a blood test). It has been suggested that those inflammatory processes can be moderated by magnesium (Tam et al. 2003). Adults who consumed less than the RDA for magnesium were as much as $75 \%$ more likely to have elevated levels of a marker of systemic inflammation (C-reactive protein) than those who consumed at least the RDA (King et al. 2005). Reducing systemic inflammatory response through weight management and dietary intervention could potentially provide a low-cost way to reduce asthma symptoms.

Obesity - a known risk factor for type 2 diabetes, heart disease and some forms of cancer - is also a risk factor for asthma, and the high energy intakes associated with overweight may increase asthma symptoms. Asthma prevalence is increased in obese people (Ford et al. 2004), and when obese asthma patients lose weight, asthma symptoms are also reduced (Shore and Fredberg 2005).

\section{Measuring magnesium and BMI}

In order to better understand the role of dietary magnesium in obese asthma sufferers, we compared magnesium 
status and dietary intake in people with asthma to healthy controls who were matched by weight and gender. Complete study details have been described elsewhere (Kazaks et al. 2006); the design and methods are briefly discussed below.

Participants. Subjects were recruited with flyers and newspaper advertisements from the general public in the Sacramento area. Based on body mass index (BMI), we matched 18 men and 36 women aged 21 to 55 years old with mild-to-moderate asthma, with 17 male and 30 female healthy control subjects. The two groups were similar in ethnic makeup, and the entire group consisted of $8 \%$ Asian, $12 \%$ black, $70 \%$ white, $12 \%$ Hispanic and 9\% undeclared individuals (the total is greater than $100 \%$ since some subjects were classified as more than one ethnicity). Written, informed consent was obtained from all participants, and the UC Davis Institutional Review Board approved the study protocol.

Procedures. At the initial screening visit, all subjects underwent a health interview and physical exam, and height and weight measurements were taken. At the second visit, all subjects were given a methacholine challenge test to determine bronchial hyperresponsiveness and diagnosis of asthma. At the third visit, all subjects were given an IV magnesium-loading test to determine their magnesium status.

On two separate visits, all subjects were asked to recall what they had eaten in the previous 24 hours, to determine their dietary magnesium intake. The interviews were done by a registered dietitian and analyzed using Food Processor software (ESHA Research, Salem, Ore.). Dietary intake was presented both as average daily magnesium and as a percentage of the magnesium RDA goal for age and gender: 400 milligrams per day (mg/day) for men and $310 \mathrm{mg} /$ day for women ages 19 to 30 , and $420 \mathrm{mg} /$ day for men and $320 \mathrm{mg} /$ day for women over age 30 (IMFNB 1997).

BMI categories. BMI was calculated as weight divided by height squared (kilograms per meters squared). Normal weight was defined as a BMI less than or equal to 24.9, overweight was a BMI between 25.0 and 29.9, and obese was a BMI greater than 30.0.

Magnesium status. Bone and muscle biopsies provide the most accurate evaluations of magnesium status. But because this is an invasive and uncomfortable procedure, we used the IV magnesium-loading test instead. Our subjects were given a magnesium infusion (0.1 millimoles magnesium per kilogram) and asked to collect urine for the following 24-hour period.

The principle of the IV magnesiumloading test is that individuals with adequate magnesium status will excrete approximately the entire amount of the infused magnesium within 24 hours.

In contrast, magnesium-depleted individuals will retain a large amount of the infused magnesium. A diagnosis of magnesium depletion is based on the percentage of magnesium retained 24 hours after the IV infusion. The normal range for IV magnesium load retention is $-39 \%$ to $3 \%$ (Rob et al. 1999).

Statistics. The results were expressed as mean \pm SEM. Data were analyzed using Student $t$-tests and analysis of variance (ANOVA). Analyses were conducted with Statview Version 5.0.1 (SAS Institute, Cary, N.C.). $P$ values $\leq 0.05$ were considered significant.

\section{Magnesium, BMI and asthma}

We found no significant differences in measures of magnesium status or magnesium intake among participants with asthma and their matched controls (table 1). The IV magnesium load retention was within the expected normal range $(-39 \%$ to $3 \%)$. Because average dietary goals differ according to age and gender, average percentage dietary magnesium goal is a more meaningful indicator of required intake than milligrams magnesium per day. Although there was a wide variation in magnesium intake, mean values showed that both the asthma and control subjects consumed less than the RDA and dietary goal for this essential mineral.

Higher BMI, lower magnesium intake. When all subjects were stratified according to BMI, there were highly
TABLE 1. Measures of magnesium status in gender- and BMI-matched control and asthma subjects*

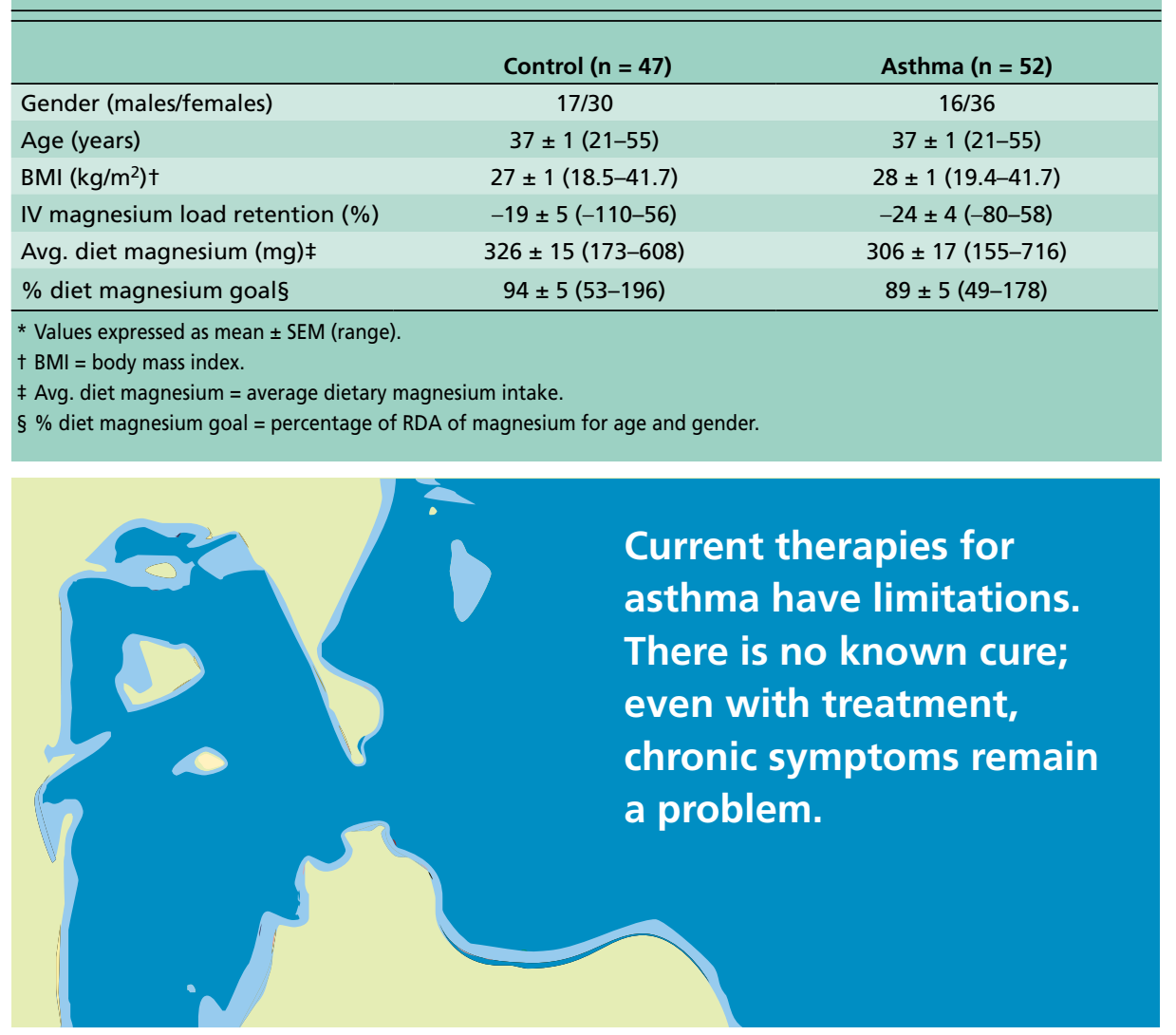


TABLE 2. Measures of magnesium status in all subjects, categorized by BMI*

\begin{tabular}{|c|c|c|c|c|}
\hline & Normal $(n=36)$ & Overweight $(n=25)$ & Obese $(n=38)$ & Pt \\
\hline IV magnesium load retention (\%) & $-38 \pm 5$ & $-17 \pm 7$ & $-10 \pm 4$ & $<0.0001$ \\
\hline Avg. diet magnesium (mg)‡ & $362 \pm 23$ & $294 \pm 16$ & $285 \pm 15$ & 0.006 \\
\hline$\%$ diet magnesium goal§ & $104 \pm 7$ & $84 \pm 5$ & $83 \pm 4$ & 0.01 \\
\hline \multicolumn{5}{|c|}{$\begin{array}{l}\text { * Mean } \pm \text { SEM. BMI = body mass index; normal } \leq 24.9 \text {; overweight }=25-29.9 \text {; obese } \geq 30 \text {. } \\
\text { † Difference between normal and obese categories. } \\
\text { ₹ Avg. diet magnesium = average dietary magnesium intake. } \\
\S \% \text { diet magnesium goal = percentage RDA of magnesium for age and gender. }\end{array}$} \\
\hline
\end{tabular}

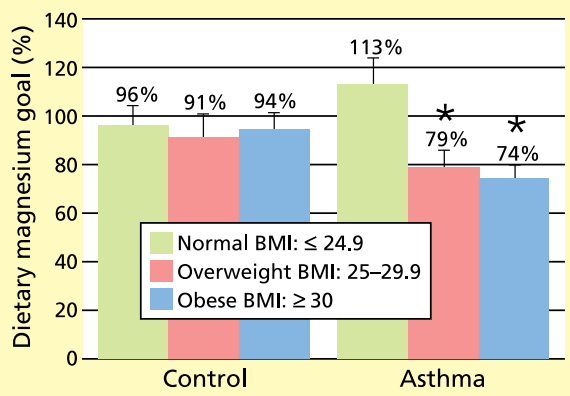

$\Delta$ Fig. 2. Percentage dietary goal for magnesium (Mg) intake in control and asthma subjects, by BMI category. Normal BMI $\leq 24.9$; overweight = 25-29.9; obese $\geq 30$. * Indicates significant difference between overweight and obese, when compared with normal category $(P \leq 0.05)$.

Fig. 3. Percentage (A) dietary goal for magnesium (Mg) intake and (B) intravenous (IV) Mg retention in control and asthma subjects, by BMI category. Normal BMI $\leq 24.9$; overweight = 25-29.9; obese $\geq 30$. * Indicates significant difference between control and asthma subjects in the obese category $(P \leq 0.05)$.

significant differences in magnesium intake and status (table 2). As BMI increased, IV magnesium load retention was higher $(P<0.0001)$, and average dietary magnesium intake $(\mathrm{P}=0.006)$ and percentage of dietary magnesium goal $(P=0.01)$ were lower.

\section{Asthma and lower magnesium} intake. When BMI categories were further divided into asthma and control groups, the control subjects had no significant differences in magnesium intake according to percentage dietary magnesium goal in any BMI category (fig. 2). However, asthma subjects had significantly lower magnesium intakes when they were overweight $(P=0.004)$ and obese $(P=0.001)$ than did normalweight asthma subjects.

Figure $3 \mathrm{~A}$ shows how percentage dietary magnesium goal varied according to BMI category when the asthma and control subjects were analyzed separately. Two-way ANOVA with

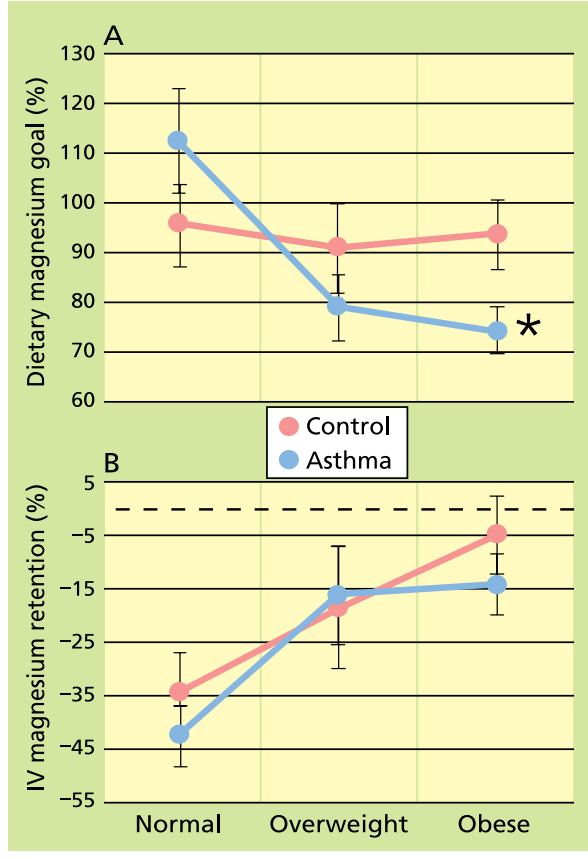

percentage dietary magnesium goal showed an interaction between BMI category and asthma or control status $(P=0.039)$, with a lower overall percentage magnesium dietary goal associated with asthma. There were no significant differences between the asthma and control subjects in the normal and overweight categories. However, the percentage dietary magnesium goal was significantly lower in obese asthma subjects than in obese controls $(P=0.023)$.

In both asthma and control participants, the percentage IV retention significantly increased as BMI category increased $(P=0.005)$ (fig. 3B).

\section{Increasing dietary magnesium}

A limitation to our study was the small sample sizes that resulted when the population was stratified. Even so, robust relationships with the BMI categories were evident. Our study also could have been strengthened by deter-

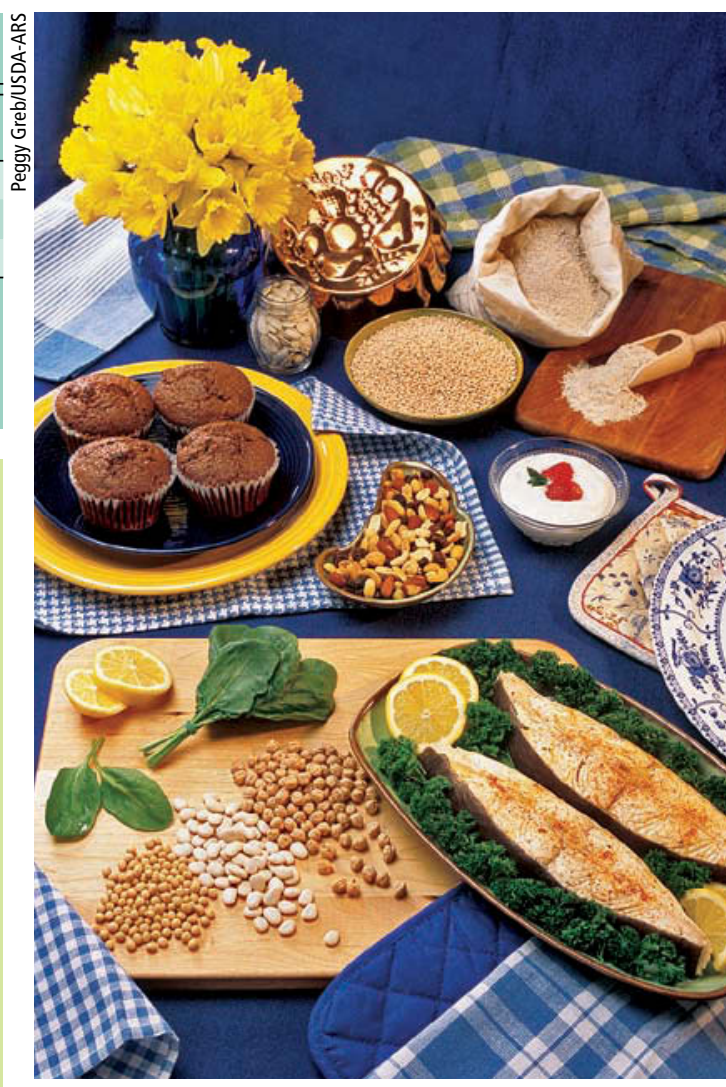

Good sources of magnesium include bran muffins, pumpkin seeds, barley, buckwheat flour, low-fat yogurt, trail mix, halibut steaks, garbanzo beans, lima beans, soybeans and spinach. Increasing dietary magnesium may reduce asthma symptoms while aiding in weight loss.

mining the income status of subjects to define how it relates to dietary magnesium and asthma. (The role of dietary magnesium supplements is the subject of another paper by the authors; in this study, subjects were limited to 50 milligrams or less per day of magnesium in dietary supplements.)

Further study is necessary to determine how people with asthma respond to the combined effects of low magnesium and increased obesity. Does inadequate magnesium intake combined with obesity increase the risk of asthma? Or does low magnesium intake combined with asthma increase obesity?

Nonetheless, this study demonstrates that people with mild-to-moderate asthma and their matched healthy controls had no differences in magnesium status or intake unless they were analyzed within BMI categories. This emphasizes that BMI must be taken into 
Asthma subjects had

\section{significantly lower} magnesium intakes when they were overweight and obese than did normalweight asthma subjects.

account to understand the biologic and behavioral influences on asthma.

Many people with asthma try complementary and alternative therapies in addition to traditional medical treatments. A 1998 survey of health care providers identified various alternative treatments used by people with asthma; dietary and nutritional approaches were the most prevalent options and were believed to be the most useful (Davis et al. 1998).

We suggest that reducing obesity and increasing magnesium-containing foods may have a dual impact on asthma control. Likewise, replacing lowmagnesium foods with high-magnesium foods may help reduce the risk of obesity in people with asthma. For most people, the best way to make sure that they get enough magnesium is to eat a wide variety of whole grains, green leafy vegetables, beans and nuts (table 3). In general, eating a varied diet that includes an abundance of fruits and vegetables also helps to prevent obesity. A combination of weight management and dietary intervention could reduce the systemic inflammatory response characteristic of asthma. Increasing dietary magnesium is a low-cost intervention that also may improve overall health.

A.G. Kazaks is Postdoctoral Researcher, Department of Nutrition, UC Davis; and J.S. Stern is Distinguished Professor, Department of Nutrition and Department of Internal Medicine, Division of Endocrinology, Clinical Nutrition and Vascular Biology, UC Davis. We wish to thank Amy Block Joy, Food Stamp Nutrition Education (FSNE) program director associated with the UC Davis Department of Nutrition, for her insights into the applications and relevance of our data for low-income populations. This study was funded in part by NIH AT00652, T32 DK 07355 and the FSNE program of California, USDA Food and Nutrition Service.

\section{TABLE 3. Selected food sources of magnesium}

\begin{tabular}{|c|c|c|}
\hline Food, standard serving* & Magnesium & Calories \\
\hline & $m g$ & \\
\hline Pumpkin and squash seed kernels, roasted, $1 \mathrm{oz}$. & 151 & 148 \\
\hline Brazil nuts, $1 \mathrm{oz}$. & 107 & 186 \\
\hline Bran, ready-to-eat cereal (100\%), 1 oz. & 103 & 74 \\
\hline Halibut, cooked, 3 oz. & 91 & 119 \\
\hline Almonds, $1 \mathrm{oz}$. & 78 & 164 \\
\hline Spinach, cooked from fresh, $1 / 2$ cup & 78 & 20 \\
\hline White beans, canned, $1 / 2$ cup & 67 & 154 \\
\hline Black beans, cooked, $1 / 2$ cup & 60 & 114 \\
\hline Tuna, yellowfin, cooked, 3 oz. & 54 & 118 \\
\hline Peanuts, dry roasted, $1 \mathrm{oz}$. & 50 & 166 \\
\hline Lima beans, baby, cooked from frozen, $1 / 2$ cup & 50 & 95 \\
\hline Soy beverage, 1 cup & 47 & 127 \\
\hline Oat bran muffin, $1 \mathrm{oz}$. & 45 & 77 \\
\hline Great northern beans, cooked, $1 / 2$ cup & 44 & 104 \\
\hline
\end{tabular}

\section{References}

[ALA] American Lung Association. 2005. Trends in Asthma Morbidity and Mortality. Epidemiology and Statistics Unit Research and Scientific Affairs. www. lungusa.org/atf/cf/\%7B7A8D42C2-FCCA-4604-8ADE7F5D5E762256\%7D/ASTHMA_TRENDS_IN_MORBIDITY_MORALITY2003.PDF (accessed May 2007).

Barnes PJ. 2004. Mediators of chronic obstructive pulmonary disease. Pharmacol Rev 56(4):515-48.

Bernstein WK, Khastgir T, Khastgir A, et al. 1995. Lack of effectiveness of magnesium in chronic stable asthma. A prospective, randomized, doubleblind, placebo-controlled, crossover trial in normal subjects and in patients with chronic stable asthma. Arch Intern Med 155(3):271-6.

Britton J, Pavord I, Richards K, et al. 1994. Dietary magnesium, lung function, wheezing, and airway hyperreactivity in a random adult population sample. Lancet 344(8919):357-62.

[CDC] Centers for Disease Control and Prevention. 2000. Self-Reported Asthma Prevalence Among Adults - United States, 2000. MMWR 50(32):682-6.

Chande VT, Skoner DP. 1992. A trial of nebulized magnesium sulfate to reverse bronchospasm in asthmatic patients. Ann Emerg Med 21(9):1111-5.

Cheuk DK, Chau TC, Lee SL. 2005. A metaanalysis on intravenous magnesium sulphate for treating acute asthma. Arch Dis Child 90(1):74-7.

Davis PA, Gold EB, Hackman RM, et al. 1998. The use of complementary/alternative medicine for the treatment of asthma in the United States. J Investig Allergol Clin Immunol 8(2):73-7.

Ford ES, Mannino DM, Redd SC, et al. 2004 Body mass index and asthma incidence among USA adults. Eur Respir J 24(5):740-4.

Hartert TV, Peebles RS. 2001. Dietary antioxidants and adult asthma. Curr Opin Allergy Clin Immunol 1(5):421-9.

Haury V. 1940. Blood serum magnesium in bronchial asthma and its treatment by the administration of magnesium sulphate. J Lab Clin Med 26:340-1.
[IMFNB] Institute of Medicine Food and Nutrition Board. 1997. Dietary Reference Intakes: Calcium, Phosphorus, Magnesium, Vitamin D and Fluoride. Washington, DC: Nat Acad Pr. 448 p.

Kazaks A, Uriu-Adams JY, Stern JS, Albertson TE. 2006. Multiple measures of magnesium status are comparable in mild asthma and control subjects. J Asthma 43(10):783-8.

King DE, Mainous AG 3rd, Geesey ME, Woolson RF. 2005. Dietary magnesium and C-reactive protein levels. J Am Coll Nutr 24(3):166-71.

Mathew R, Altura BM. 1991. The role of magnesium in lung diseases: Asthma, allergy and pulmonary hypertension. Magnes Trace Elem 10(2-4):220-8.

[NHLBI] National Heart, Lung, and Blood Institute. 2002. NAEPP Expert Panel Report: Guidelines for the Diagnosis and Management of Asthma; Update on asthma/index.htm (accessed May 2007).

NHLBI. 2006. What Is Asthma? www.nhlbi.nih gov/health/dci/Diseases/Asthma/Asthma_Whatls.html (accessed May 2007)

Rob PM, Dick K, Bley N, et al. 1999. Can one really measure magnesium deficiency using the 246(4):373-8.

Rolla G, Bucca C, Arossa W, Bugiani M. 1987. Magnesium attenuates methacholine-induced bronchoconstriction in asthmatics. Magnesium 6(4):201-4.

Shore SA, Fredberg JJ. 2005. Obesity, smooth muscle, and airway hyperresponsiveness. J Allergy Clin Immunol 115(5):925-7.

Tam M, Gomez S, Gonzalez-Gross M, Marcos A. 2003. Possible roles of magnesium on the immune system. Eur J Clin Nutr 57(10):1193-7.

[USDA] US Department of Agriculture. 2006 USDA National Nutrient Database for Standard Reference, Release 19. Nutrient Data Laboratory, Agricultural Research Service. www.ars.usda.gov/ba/ bhnrc/ndl (accessed May 2007). Selected Topics 2002. www.nhlbi.nih.gov/guidelines/ short-term magnesium loading test? J Intern Med 